\title{
Knowledge in the model of dynamics and stability of an industrial enterprise
}

\author{
Sergey Chuprov ${ }^{1, *}$ \\ ${ }^{1}$ Baikal State University, 11, Lenin Str., Irkutsk, Russia
}

\begin{abstract}
The formation and development of the knowledge economy logically lead to the need for an indepth study of the nature and properties of information in economic systems. The priority value of the information resource and knowledge in the activities of industrial enterprises is determined both by the prospects for the development of science-intensive products and their competitiveness in the world and domestic markets, and by the desire to ensure the stable operation of the enterprise in a highly disturbed business environment. With the search and analysis of the dependences of the production, financial and economic processes taking place in them on the control information, the systemic interaction of the enterprise resources and the dynamics of key indicators of the activity of industrial enterprises are revealed.From the standpoint of thermodynamics, statistical physics and cybernetics, it is proposed to spread the information concept of V. Trapeznikov to substantiate and modelling the dependence of a number of economic and financial indicators on the amount of accumulated information in the controlled complex of an industrial enterprise.Modeling and interpretation of the relationship between the volume of control information with the uncertainty of the functioning of an industrial enterprise and the achieved level of knowledge about it is carried out. The condition for the stability of the indicator of the effect of activity and the financial stability of the enterprise (autonomy ratio) is formulated. The mathematical condition for the range of changes in the level of knowledge of industrial enterprises has been substantiated.
\end{abstract}

Keywords: entropy, knowledge, industry, information, model, stability

\section{Introduction}

The harmonization of financial and economic processes in industry is under the increasing influence of the flow of information, the resource of which becomes dominant to ensure favorable conditions for their stability and strengthen competitive positions. The painful institutional transformations experienced by the Russian industry under the pressure of a powerful stream of indignations prompt analysts to investigate not only the nature and dominant trends of the business environment, but also to form the mechanism of adaptive management of domestic enterprises. At the same time, the high dynamism of the outraged environment and the mastery of the tools for managing the development of enterprises in the globalizing world reasonably concentrate the efforts of enterprise managers on balancing financial proportions and increasing the economic efficiency of industrial enterprises as part of their intensive innovative modernization.

The relevance of understanding information and financial and economic interaction in the activities of an enterprise increases even more if we take into account the fact that the dependence of the effective and solvent activities of enterprises on the volume of accumulated information has not yet found deep theoretical and methodological study. The significance of the scientific results published by analysts, both in conceptual and applied aspects, is higher for understanding the nature and dynamics of communication of information and financial and economic processes at industrial enterprises in the context of innovative development of enterprises $[1,9,11,14]$.

\section{Problem Statement}

The modern knowledge economy is being formed in the space of world challenges and threats, saturated with a stormy stream of technological innovations that are fundamentally transforming the face of industrial production. Aggravating and motivating indignations excite the business environment and dictate the need for a radical modernization of economic structures and the widespread development of science-intensive means of manufacturing products and providing services. Organizational, managerial, design and technological innovations now not only determine the competitiveness of manufactured products, but also, with the increase in information and innovation resources of enterprises, strengthen the stability of their activities in an outraged competitive environment.

\footnotetext{
* Corresponding author: ChuprovSV@yandex.ru
} 
The full use and involvement of useful information in management loops and production processes was objective and did not meet any conceptual objections, since they served to revolutionize the development of the technical base of the enterprise, optimize product output and achieve targets due to improved coordination of actions of all its departments. After all, the progressiveness of technological methods of manufacturing products and rendering services, and the quality of management decisions are ultimately determined by the valuable information contained in them. At the same time, the postulate of an innovative economy, recognized in principle, needs to deepen theoretical concepts, search and systematic understanding of the relationship of a complex of resources from the point of view of the impact of information on the structural and behavioral attributes of an industrial enterprise.

\section{Research Questions}

Within the research task set, the following questions are subject to studying:

1. Justification of the application of theoretical approaches to solving the problem of analyzing the influence of information on the performance of an enterprise.

2. Formalization and analysis of the model of the dependence of the indicators of the effect of the enterprise on the size of the information resource available to it.

3. Determination of the dependence and interpretation of the model of the dynamics of financial and economic indicators and the stability of the enterprise on its level of knowledge.

\section{Purpose of the Study}

The purpose of the study is to clarify the nature of the information impact on the activities of an industrial enterprise, to assess and analyze the impact of the level of knowledge on economic dynamics and the stability of its functioning.

\section{Research Methods}

For the research, the following methods were used: thermodynamics and statistical physics, cybernetics and information theory.

\section{Findings}

Information theory draws its postulates from the basic concepts of thermodynamics and statistical physics about the behavior of macroscopic physical systems that are in a state of equilibrium and pass from it to other states. The fundamental studies of R. Clausius [7], L. Boltzmann [5], J. Gibbs [8], M. Smoluchowski [7] created and developed the science of the nature and laws of thermodynamic processes, which are quite universal for a wide class of systems. Their dynamic picture is characterized by a measure of the probability of a certain state of a macroscopic system, or its entropy, In particular, a closed system left to itself over time irreversibly comes to an equilibrium state with maximum entropy.

The organic relationship of entropy, organization and the amount of information in the system was analyzed by the classics of cybernetics. The organization of a system, N. Wiener believed, is the amount of information in it, while the entropy of a system is a measure of its disorganization [16]. Such an interpretation of the attributes of a dynamical system was found logical and was successfully developed in the works by L. von Bertalanffy [4], S. Beer [3], L. Brillouin [6], C. Shannon [13], W. Ashby [2] et al.

Statistical physics not only linked the entropy of a system with the probability of its macroscopic state, but also takes into account fluctuations in which systems move from more probable states to less probable ones when the entropy of the system decreases [12]. Such an interpretation of entropy served to introduce the concept of information entropy as an estimate of the uncertainty of a message described by a set of quantities with the corresponding probabilities of their appearance in it. In C. Shannon's information theory [13], communication channels are stochastic and can also be characterized by the uncertainty of behavior, and its decrease is perceived by obtaining information about the functioning of the system.

If the initial entropy $H *$ of the system in the process of observing it decreases to the value $H$, the amount of information extracted in this case is equal to the difference between them:

$$
H_{*}-H=I .
$$

For an economic system with its inherent disorder of behavior to one degree or another (disruptions in the supply or movement of resources, equipment failures, mismatch of personnel actions, violation of executive discipline, downtime, technological defects, etc.), saturation with control information improves the neutralization or localization of interference. It also extinguishes abnormal disturbances and thereby increases the coordination of personnel activities and leads to an increase in the effect of the system functioning. In the statistical interpretation, the model of the dependence of the effect $E$ of the functioning of the system on the amount of the information $I$ entered into it is expressed by the formula obtained by $\mathrm{V}$. Trapeznikov [15]:

$$
\frac{E}{E_{\max }}=1-e^{-\frac{I}{I_{0}}},
$$

where $E_{\max }-$ effect of a perfectly functioning system (maximum possible effect),

$I_{0}$ - amount of information typical for a given control object. 
The exponential dependence of the effect of the functioning of the system (productivity, income of the enterprise, etc.) on the amount of control information received into it states the fundamental feature of the economic dynamics of the activity of an industrial enterprise with different rates of increase in the effect. At first, it is almost proportional to the amount of information entered, but after the region of the greatest curvature of the exponent, it rapidly decreases, and therefore the level of the effect in the region of its small values requires less increment of control information, in contrast to the field of large values. Moreover, as the effect level approaches the maximum possible $\left(E \rightarrow E_{\max }\right.$ or $\frac{E}{E_{\max }} \rightarrow 1$ )the amount of additional input control information required at the enterprise is rapidly increasing, as a result of which a balanced approach to reducing entropy in the activity of an industrial enterprise and investments to maximize its effect is justified.

In the development of this conclusion, we will find the condition for its stability, bearing in mind the change in the magnitude of the effect in the noncritical range when the effect of the disturbances destabilizing its functioning on the enterprise. Let us assume that the relative indicator $\frac{E}{E_{\max }}$ stability will be inherent if the value of this fraction, despite the influence of harmful disturbances, remains in the permissible range of values from the lower $K_{e}^{l}$ to the upper $K_{e}^{u}$ boundary.

With the transformation of formula (1), we find the required condition for the stability of the indicator of the effect of the enterprise:

$$
\ln \left(1-K_{e}^{l}\right)^{-1} \leq \frac{I}{I_{0}} \leq \ln \left(1-K_{e}^{u}\right)^{-1}
$$

This equality implies the expected economic dynamics: ensuring the stability of the effect size in the area of its small values is less resource-intensive than in the area of large values. For example, comparing ranges of the same length, but at different points on the number axis, we make sure that when moving from the allowable range $0.4 \ldots 0.5\left(0,4 \leq \frac{E}{E_{\max }} \leq 0,5\right)$ to the "far" range $0,8 \ldots 0,9$, it is required to ensure that the amount of control information is entered into the enterprise by more than 3 times (for lower limits of 3.16 and upper limits of 3.33 times). And this entails the attraction of additional resources for the collection, transmission, processing and storage of information arrays, the complication of the enterprise management system with equipping it with innovative computer technologies.

In terms of the relative value of the economic effect $\frac{E}{E_{\max }}$, we summarize that it can be used to judge the degree of suppression of disturbances, increase in orderliness (decrease in entropy) in the functioning of the enterprise, and, therefore, the achieved quality of its management. In the concept of V. Trapeznikov [15], ratio $\frac{E}{E_{\max }}$ is determined by the amount of information entered and its usefulness and assesses the level of perfection in managing the economic system $L_{m}$, reflecting the effectiveness of the activities of its management personnel.

Let us formalize the dependence of the amount of control information $I$ in the economic system on the level of perfection of its control $L_{m}$. Omitting the mathematical calculations, let us give the final form of the formula:

$I=I_{0} \ln \left(\frac{B_{0}}{1-L_{m}}\right)$,

where $B_{0}$ - disorder of the system in the initial state.

From the point of view of the analysis of economic dynamics, it is confirmed that the amount of information entered into the system $I$ is logarithmically related to its disorder $B_{0}$ and the level of perfection of system management $L_{m}$. In an effort to ensure an increase in the latter, it is easier to achieve an increase $I$ if the disorder in the system $B_{0}$ is sufficiently low. Conversely, when $B_{0}$ it is quite large, it is more difficult to increase the level $L_{m}$, since to decrease the entropy it is necessary to increase the amount of information $I$.

According to formula (2), if the amount of control information $I$ in the economic system is equal to the initial $I_{0}$, then with the initial disorder $B_{0}=0.50$ level of perfection of system management $L_{m}=0.82$, and at higher disorder $B_{0}=0.75$ value $L_{m}$ below $\left(L_{m}=0.72\right)$. With increasing control information $I$ the discrepancy between the values $L_{m}$ at different $B_{0}$ decreases. So, if in the economic system the amount of control information $I$ is 5 times more than it was before its receipt $\left(I=5 I_{0}\right)$, then with the same $B_{0}(0.50$ and 0.75$)$ the values $L_{m}$ match up to hundredths (0.99).

Meanwhile, it is obvious that the successful financial and economic activity of an industrial enterprise is based not only on circulating control information, but also on the competent use of the knowledge accumulated by society, materialized in the technical base of the enterprise (designs of machines, units, equipment, their complexes, etc.), which determine the level of perfection of the system $L_{s}$. The more modern science-intensive design and technological solutions are embodied in the production equipment of an enterprise, the higher its capabilities in manufacturing competitive products, adaptability and stability in the business environment. Aggregate indicator combining levels $L_{m}$ and $L_{s}$, according to V. Trapeznikov [15], characterizes the level of knowledge and skills used, or in short, the level of knowledge $L_{k}$, thus mathematically there is a work $L_{k}=L_{m} L_{s}$.

The level of knowledge $L_{k}$ is affected by the influence of labor (qualifications and skills of workers), 
social and psychological (dedication, persistence of workers, discipline, etc.) factors. In formal terms, the value $L_{k}$ is proportional to the multiplication of labor productivity $P_{l}$, measured by the generated net product, and capital productivity $P_{c}$. Since the coefficient introduced into the definition formula $L_{k}$, taking into account industry conditions, for the country's economy and most industries is taken equal to 1.0 , we can write an expression for calculating the level of knowledge: $L_{k}=P_{l} P_{c}$ (ruble/man).

Now we will focus on the formalization and analysis of the relationship between the level of knowledge accumulated in the economic system of an industrial enterprise, with its characteristics of work efficiency and financial stability, which allows us to find a remarkable economic dynamics of the enterprise.

To do this, we will use the author's proposed model of dependence of one of the main indicators of the financial stability of the enterprise- its autonomy ratio on a number of indicators of the enterprise functioning:

$$
K_{a . r}=L_{k} \frac{K_{f . w . c}}{P_{l} E_{o . f}},
$$

where $K_{a . r}$ - autonomy ratio,

$L_{k}$ - the level of knowledge at the enterprise,

$K_{\text {f.w.c }}$ - the share of fixed assets and standardized working capital in the value of the property of the enterprise,

$P_{l}-$ labor productivity, measured by the generated net product,

$E_{o . f}$ - efficiency (turnover) of own funds (the ratio of proceeds from product sales to the cost of the company's own funds).

Despite the direct dependence of the autonomy ratio $K_{a . r}$ on the level of knowledge $L_{k}$, in reality, the tendency to change their values was predominantly opposite (only occasionally did they have a unidirectional change) due to the fact that the increase in labor productivity $P_{l}$ was very noticeable and was accompanied by a relatively low unevenness of indicators efficiency (turnover) of own funds $E_{o . f}$ and the share of fixed assets and standardized working capital $K_{f . w . c}$. At the same time, the indicators $P_{l}$ and $E_{o . f}$ experienced the positive impact of increasing the level of knowledge $L_{k}$ (saturation with control information, accompanied by the buildup of hardware and software products for management purposes) of the enterprise management system, and $K_{a . r}$ the restraining effect of the accumulation of short-term liabilities.

Along with this, in order to clarify the dynamics of the magnitude of the level of knowledge $L_{k}$, it is pertinent to ask about the extent to which it varies. Taking into account that, by definition, the values of the coefficients are $K_{a . r} \leq 1.0$ and $K_{\text {f.w.c }} \leq 1.0$, simple mathematical transformations lead to an inequality that sets the limits of change in the level of knowledge $L_{k}$ :

$$
K_{a . r} P_{l} E_{o . f} \leq L_{k} \leq \frac{P_{l} E_{o . f}}{K_{f . w . c}} .
$$

It shows that at the achieved values $P_{l}$ and $E_{o . f}$, the lower the limit of the level of knowledge $L_{k}$ at an industrial enterprise is determined by the coefficient $K_{a . r}$, and the upper limit - by the coefficient $K_{f . w . c}$. Thus, the characteristic of the financial stability of the enterprise $K_{a . r}$, which summarizes the effectiveness of management activities, the structure and return of the enterprise's resources, sets the minimum level of knowledge $L_{k}$. At the same time, its maximum is fixed by the coefficient $K_{\text {f.w.c }}$, while the smaller it is, the further the upper limit of the value $L_{k}$ is pushed back and the range for increasing the level of knowledge expands. Thus, thanks to skillful management and filling with useful information, an industrial enterprise manages to increase the effect of its activities with a decreasing share of fixed assets and normalized working capital, expressed by the coefficient $K_{\text {f.w.c. }}$

\section{Conclusion}

The phenomena of global challenges and threats, accelerated by the rapid development of high-tech production, give rise to extraordinary dynamic processes in the space of national economies, prompting industrial enterprises to adapt to the stunning disturbances of the business environment. In view of this, embedding them in an innovative economy and gaining stability demanded both the search and implementation of modern science-intensive technologies and widespread use in scientific research of the symbiosis of natural and social branches of knowledge.

The growing influence of an information resource on increasing the competitiveness and efficiency of industrial enterprises logically leads to the need to understand and apply universal laws of behavior of macroscopic systems. Revealing the features of the economic dynamics of enterprises, they enrich the theoretical concepts, methods and models of analysis and information support of the effect of enterprise functioning. Drawing on the views of thermodynamics, statistical physics, cybernetics and information theory, it becomes possible to give an entropic interpretation to the information resource of an enterprise and to develop the approach of $\mathrm{V}$. Trapeznikov to the relationship of entropy, information and the effect of the enterprise.

The analytical value of the approach and model proposed in the article lies in the fact that they allow one to assess the measure and understand the nature of the influence of the information resource and the level of knowledge on the economic dynamics and stability of the efficient operation of an industrial enterprise.

\section{References}

1. B.Y. Akcali, E. Sismanoglu. Innovation and the Effect of Research and Development (R\&D) Expenditure on Growth in Some Developing and Developed Countries. Proc. - Soc. and Behav. Sci., 195, 768-775 (2015)

2. W.R. Ashby. An Introduction to Cybernetics (Foreign liter. Publ., Moscow, 1959) 
3. S. Beer. Cybernetics and Management (Sci. Publ., Moscow, 1965)

4. L. von Bertalanffy. General system theory - a critical review. Studies on the General Systems Theory (Progress Publ., Moscow, 1969)

5. L. Boltzmann. Articles and Speeches (Sci. Publ., Moscow, 1970)

6. L. Brillouin. Scientific Uncertainty and Information (Mir Publ., Moscow, 1966)

7. S. Carnot, W. Thomson (Kelvin), R. Clausius, L. Boltzmann, M. Smoluchowski, A. Timiryazev (Ed.). The Second Law of Thermodynamics (LKI Publ., Moscow, 2007)

8. J. Gibbs. Thermodynamics. Statistical Mechanics (Sci. Publ., Moscow, 1982)

9. M.J. Hashim, I. Osman, S.M. Alhabshi. Effect of Intellectual Capital on Organizational Performance. Proc. - Soc. and Behav. Sci., 211, 207-214 (2015)

10. R. Kamasa. Determinants of innovation performance: A resource-based study. Proc. - Soc. and Behav. Sci., 195, 1330-1337 (2015)

11. L. Márquez-Ramos, I. Martínez-Zarzoso. The Effect of Technological Innovation on International Trade. A Nonlinear Approach. Open-Assessm. E-J., 4, 1-37 (2010)

12. A.M. Prokhorov (Ed.). Physical encyclopedic dictionary (Sov. Encycl., Moscow, 1983)

13. C. Shannon. The Mathematical theory of Communication (The Univ. of Illinois Press, Urbana, Illinois, 1949)

14. G.L. Sipos, A. Ionescu. The Innovation - Corporate Performance Relationship in Emergent Countries. Proc. - Soc. and Behav. Sci., 238, 124-132 (2018)

15. V.A. Trapeznikov. Management and scientific and technological progress (Sci. Publ., Moscow, 1983)

16. N. Wiener. Cybernetics or Control and Communication in the Animal and the Machine (Sci. Publ., Moscow, 1983) 